Allgemeinheit dienende Maßnahmen des Hochwasserschutzes - und damit auch für die Errichtung von gesteuerten Flutpoldern - Anwendung finden kann (vgl. zur baurechtlichen Veränderungssperre nach $\iint 14 \mathrm{ff}$. BauGB Breuer/Gärdiz, Öffentliches und privates Wasserrecht, Rdnr. 1325; vgl. auch Appel in Berendes/Frenz/Müggenborg, WHG, $\$ 86$ Rdnr. 2 m. w. N.; Hünnekens in Landmann/Rohmer, Umweltrecht, \$76 WHG Rdnr. 35). Auch dieses Planungssicherungsinstrument kann im Einzelfall dem Schutz einer ernsthaften und konkreten Planung dienen und sicherstellen, dass sich das in Rede stehende wasserwirtschaftliche Vorhaben in der in $\$ 86$ Abs. 3 WHG vorgesehenen maximalen Sperrdauer von vier Jahren überhaupt realisieren lässt (vgl. Appel in Berendes/Frenz/Müggenborg, a.a.O., Rdnr. 19 m.w. N.). Das Vorhandensein eines zusätzlichen Sicherungsinstruments erscheint aber, nicht zuletzt angesichts der erheblichen Bedeutung der Belange des Hochwasserschutzes, nicht systemwidrig. Es liegen auch keine Anhaltspunkte dafür vor, $\$ 86 \mathrm{WHG}$ insofern als abschließend anzusehen. Zudem sieht die vorläufige Sicherung nach $\$ 76$ Abs. 3 WHG i. V.m. Art. 47 BayWG gemäß Art. 47 Abs. 4 BayWG nicht nur einen Sicherungszeitraum von (lediglich) drei Jahren mit einer Verlängerungsmöglichkeit um ein Jahr vor ( $\$ 86$ Abs. $3 \mathrm{WHG}$ ), sondern eine Zeitdauer von fünf Jahren mit der Möglichkeit, diesen in begründeten Einzelfällen um bis zu zwei Jahre zu verlängern.

Hinzu kommt die Gesetzeshistorie. Der Gesetzgeber begründete die Einführung der im Wesentlichen in \$86 WHG inhaltsgleich übernommenen Vorgängervorschrift des $\$ 36$ a WHG i.d.F. des Vierten Gesetzes zur Änderung des Wasserhaushaltsgesetzes vom 26.4.1976 (BGB1. I S. 1109) mit der Notwendigkeit, schon im Planungsstadium die Flächen für raumbeanspruchende Maßnahmen, die im Allgemeinen mehrjähriger Planungsvorbereitungen bedürfen, von Veränderungen, die die Vorhaben stören oder vereiteln könnten, freizuhalten, weil diese weitgehend ortsgebunden sind und die nach der Planung vorgesehenen Standorte sich im allgemeinen nicht oder nur unter größten Schwierigkeiten ändern lassen (vgl. BT-Drs. 7/888 S. 20). Trotz des Vorhandenseins dieses Instruments hat der Normgeber mit der Schaffung des $\$ 31 \mathrm{~b}$ Abs. 5 WHG i.d.F. des Gesetzes zur Verbesserung des vorbeugenden Hochwasserschutzes vom 3.5.2005 (BGBl. I S. 1224) die spezielle Möglichkeit der vorläufigen Sicherung von Überschwemmungsgebieten zusätzlich geschaffen (kritisch hierzu ausdrücklich unter Verweis auf $\$ 36$ a WHG: Drost, Das Wasserrecht in Bayern, Stand Juli 2009, \$31b WHG Rdnr. 98; danach war die Notwendigkeit der Regelung in $\$ 31$ b Abs. 5 WHG im Laufe der parlamentarischen Beratungen umstritten, was in den Gesetzesmaterialien jedoch keinen Niederschlag gefunden hat). Damit hat der Gesetzgeber zu erkennen gegeben, dass diese Bestimmung neben der im damaligen \$36a WHG geregelten Veränderungssperre Anwendung finden kann. Da im Rahmen dieses Gesetzgebungsverfahrens nicht nur \$31b Abs. 5 WHG neu eingeführt, sondern im Hinblick auf $\$ 36$ a WHG auch eine Anpassung an das ROG vorgenommen wurde (vgl. BT-Drs. 15/3168 S. 15), ist sichergestellt, dass der Normgeber beide Bestimmungen im Blick hatte. Es spricht daher alles dagegen, $\$ 86 \mathrm{WHG}$ als insofern abschließende Regelung zu verstehen, die eine Heranziehung des $\$ 76$ Abs. 3 WHG sperrt (vgl. Appel in Berendes/ Frenz/Müggenborg, WHG, \86 Rdnr. 89; Czychowski/ Reinhardt, WHG, $\$ 86$ Rdnr. 25 jeweils zum Verhältnis der Veränderungssperre nach $\$ 86$ WHG zu nach anderen Gesetzen bestehenden Veränderungssperren und sonstigen Nutzungsbeschränkungen). Die nähere Ausgestaltung des Verfahrens zur vorläufigen Sicherung und Festsetzung eines Überschwemmungsgebiets überlässt $\$ 76$ Abs. 2 WHG dem Landesgesetzgeber (Czychowski/Reinhardt, WHG, \$76 Rdnr. 16). Da nach Art. 47 Abs. 2 Satz 2 BayWG die vorläufige Sicherung durch gesetzliche Fiktion bewirkt wird, wird hierbei der Verwaltungsaufwand, wie er bei Erlass ei- ner gesondert zu erlassenden Veränderungssperre erforderlich ist, vermieden (Drost, das neue Wasserrecht in Bayern, Stand März 2019, Art. 47 BayWG Rdnr. 4). Dies dient der Effektivierung des Hochwasserschutzes, die angesichts der Bedeutung dieser Belange keinen Bedenken begegnet.

Etwas anderes ergibt sich auch nicht aus dem Umstand, dass die Veränderungssperre, wie bereits oben ausgeführt, regelmäßig nach drei Jahren bzw. bei Verlängerung oder Erneuerung nach Ablauf eines weiteren Jahres außer Kraft tritt (\$86 Abs. 3 Satz 1 und 2 WHG), während die vorläufige Sicherung von Überschwemmungsgebieten nach maximal sieben Jahren endet (Art. 47 Abs. 4 Satz 2 und 3 BayWG). Der Einwand des Klägers, eine „Hintereinanderschaltung“ von vorläufiger Sicherung und Veränderungssperre hätte eine entschädigungslose Eigentumsbindung für den Eigentümer der gesicherten Fläche für einen Zeitraum von bis zu 11 Jahren zur Folge, wäre gegebenenfalls in einem Verfahren gegen eine der vorläufigen Sicherung nachfolgende Veränderungssperre zu berücksichtigen (vgl. zur Frage der Anrechnung anderer Veränderungssperren auf die Dauer der Veränderungssperre nach $\$ 86$ WHG Schenk in Sieder/Zeitler/Dahme, WHG und AbgrG, \$86 Rdnr. 34). Das Vorhandensein mehrerer Sicherungsinstrumente, die jeweils zeitlichen Höchstgrenzen unterworfen sind, berechtigt nicht automatisch dazu, von beiden Möglichkeiten beim selben Vorhaben Gebrauch zu machen und die vorgesehene zeitliche Begrenzung dadurch auszudehnen.

https://doi.org/10.1007/s10357-020-3702-5

\section{Wegen Befreiung von den Verboten einer Land- schaftsschutzverordnung $u$. a. für den Neubau und die Modernisierung der Freianlagen auf der Insel Grafenwerth hier: Regelung der Vollziehung}

BNatSchG §14 Abs. 1, §15 Abs. 1, 2 und 5, §17 Abs. 1, §34 Abs. 1, §67 Abs. 1; UmwRG §1 Abs. 1, §2 Abs. 1 und 4, §3, $\S 7$ Abs. 5; VwGO §80 Abs. 2, 3 und 5, §80a Abs. 3

1. Hinreichende inhaltliche Bestimmtheit von Befreiungen von Verboten einer Landschaftsschutzgebietsverordnung setzt voraus, dass insbesondere für den Adressaten des Verwaltungsakts die von der Behörde getroffene Regelung so vollständig, klar und unzweideutig erkennbar ist, dass er sein Verhalten danach ausrichten kann.

2. Im Einzelnen richten sich die Anforderungen an die notwendige Bestimmtheit nach den Besonderheiten des jeweils anzuwendenden und mit dem Verwaltungsakt umzusetzenden materiellen Rechts, vgl. BVerwG, Beschl. v. 9.10.2012 - 7 VR 10.12, juris, Rdnr. 10.

3. Die Erteilung einer Befreiung setzt eine atypische Sondersituation voraus, die der Verordnungsgeber beim Erlass der Verordnung nicht in den Blick genommen hat. Eine Befreiung kommt nur in Betracht, wenn die Anwendung der Ge- oder Verbotsnorm im Einzelfall zu einem Ergebnis führen würde, das dem Normzweck nicht mehr entspricht und deshalb normativ so nicht beabsichtigt ist.

- Leitsätze der Redaktion -

VG Köln, Beschluss vom 22. 5. 2020 - 14 L 202/20-

Der Antrag hat in dem aus dem Tenor ersichtlichen Umfang Erfolg. Der sinngemäß gestellte Antrag, die aufschiebende Wirkung der Klage 14 K 466/20 gegen den Bescheid des Antragsgegners vom 20.1.2020 wiederherzustellen, ist zulässig. Die Klage des Antragstellers hat keine aufschiebende Wirkung, nachdem der Antragsgegner mit Bescheid vom 31. 1. 2020 nach $₫ 80$ Abs. 2 Satz 1 Nr. 4 VwGO die sofortige Vollziehung des angegriffenen Bescheids angeordnet hat. Der Antragsteller ist eine anerkannte Umweltvereinigung im Sinne des $\$ 3$ UmwRG und nach $\$ 2$ Abs. 1 
UmwRG antragsbefugt Die von dem Antragsgegner zu Gunsten der Beigeladenen erteilte Befreiung von den Verboten der Ordnungsbehördlichen Verordnung über die Landschaftsschutzgebiete in den Städten Königswinter und Bad Honnef im Rhein-Sieg-Kreis vom 31.8.2006 (im Folgenden: Landschaftsschutzgebietsverordnung - LSGVO) ist ein Verwaltungsakt im Sinne des $\$ 1$ Abs. 1 Satz 1 Nr. 5 UmwRG, durch den ein in $\$ 1$ Abs. 1 Satz 1 Nrn. 1 bis 2b UmwRG nicht genanntes Vorhaben unter Anwendung umweltbezogener Vorschriften zugelassen wird.

\section{Aus den Gründen:}

Der Antrag ist begründet, soweit der angegriffene Bescheid das Vorhaben der Beigeladenen in den Bauabschnitten 2 und 3 betrifft (unten I.), im Übrigen, d.h. hinsichtlich des Bauabschnitts 1, ist der Antrag unbegründet (unten II.). Der Bauabschnitt 1 umfasst die Nordspitze der Insel und reicht im Süden bis an die Zufahrtstraße der nördlichen Brücke. Der Bauabschnitt 2 beinhaltet die Promenade am Westufer vom Restaurant bis zum Freibadeingang und der Bauabschnitt 3 die Zufahrtstraße der nördlichen Brücke und das Umfeld des Mineralbrunnens.

I. Das Gericht der Hauptsache kann gemäß $₫ 80$ Abs. 5 Satz 1 zweite Alternative i.V.m. \$80a Abs. 3 Satz 2 VwGO auf Antrag die aufschiebende Wirkung eines Rechtsbehelfs ganz oder teilweise wiederherstellen. Dabei trifft das Gericht auf Grund der sich im Zeitpunkt seiner Entscheidung darstellenden Sach- und Rechtslage eine Ermessensentscheidung darüber, ob die Interessen, die für einen sofortigen Vollzug des angefochtenen Verwaltungsakts sprechen, oder diejenigen, die für die Anordnung der aufschiebenden Wirkung streiten, höher zu bewerten sind. Wesentliches Element dieser Interessenabwägung ist die Beurteilung der Erfolgsaussichten des Rechtsbehelfs in der Hauptsache, die dem Charakter des Eilverfahrens entsprechend nur aufgrund einer summarischen Prüfung der Sach- und Rechtslage erfolgen kann. Ein Rechtsbehelf einer anerkannten Umweltvereinigung gegen eine Entscheidung im Sinne des $\$ 1$ Abs. 1 Satz $1 \mathrm{Nr}$. 5 UmwRG ist begründet, soweit diese gegen umweltbezogene Vorschriften verstößt, die für die Entscheidung von Bedeutung sind, und der Verstoß Belange berührt, die zu den Zielen gehören, die die Vereinigung nach ihrer Satzung fördert, $\$ 2$ Abs. 4 Satz 1 UmwRG.

Ausgehend hiervon fällt die lnteressenabwägung $\mathrm{zu}$ Gunsten des Antragstellers aus, soweit der angegriffene Bescheid das Vorhaben der Beigeladenen in den Bauabschnitten 2 und 3 betrifft. Die Klage in der Hauptsache wird insoweit mit hoher Wahrscheinlichkeit zumindest zu der Feststellung führen, dass der Bescheid rechtswidrig ist.

Gegenstand des angegriffenen Bescheids ist das Vorhaben der Beigeladenen, die Freianlagen auf der Insel Grafenwerth zu modernisieren und neu anzulegen, so wie es in dem Landschaftspflegerischen Begleitplan vom 19.6.2019 und ergänzend in der Artenschutzprüfung Stufe II dargestellt ist. Die im Bauabschnitt 2 des Vorhabens geplanten (Bau-)Maßnahmen werden in den genannten Unterlagen ausreichend detailliert beschrieben, sodass der angegriffene Bescheid insoweit hinreichend bestimmt ist. Ob dies auch für den Bauabschnitt 3 zutrifft, lässt die Kammer offen (unten 1.). Die tatbestandlichen Voraussetzungen der erteilten naturschutzrechtlichen Befreiung liegen für die Bauabschnitte 2 und 3 des Vorhabens nicht vor (unten 2.). Der Antragsteller kann den dadurch begründeten Verstoß gegen umweltbezogene Vorschriften rügen. Ob in der Hauptsache die angegriffene Befreiung aufzuheben oder nur ihre Rechtswidrigkeit festzustellen sein wird, kann im Verfahren des vorläufigen Rechtsschutzes offen bleiben (unten 3.) Der Eingriffsgenehmigung fehlt damit die Grundlage, sodass der Antrag auch insoweit Erfolg hat (unten 4.).

1. Die Befreiung von den Verboten der Landschaftsschutzgebietsverordnung ist hinsichtlich des Bauabschnitts 2 inhaltlich hinreichend bestimmt, $₫ 37$ Abs. 1 VwVfG NRW.

Hinreichende inhaltliche Bestimmtheit setzt voraus, dass insbesondere für den Adressaten des Verwaltungsakts die von der Behörde getroffene Regelung so vollständig, klar und unzweideutig erkennbar ist, dass er sein Verhalten danach ausrichten kann. Es reicht aus, wenn sich die Regelung aus dem gesamten Inhalt des Bescheids, insbesondere seiner Begründung, sowie den weiteren den Beteiligten bekannten oder ohne Weiteres erkennbaren Umständen unzweifelhaft erkennen lässt. Im Einzelnen richten sich die Anforderungen an die notwendige Bestimmtheit nach den Besonderheiten des jeweils anzuwendenden und mit dem Verwaltungsakt umzusetzenden materiellen Rechts, vgl. BVerwG, Beschl. v. 9. 10.2012 - 7 VR 10.12, juris, Rdnr. 10; und Urt. v. 20.4.2005 - 4 C 18.03, juris, Rdnr. 53.

Bei einer Genehmigung muss eindeutig sein, was genau genehmigt wurde und welchen Umfang die gestattende Wirkung der Genehmigung hat. Bestimmbarkeit reicht hierbei aus. Das Gewollte kann sich auch aus der Bezugnahme auf bestimmte Antragsunterlagen ergeben, vgl. BVerwG, vom 20.5.2014 - 4 B 21.14, juris, Rdnr. 9; OVG Münster, Beschl. v. 22. 5.2015- 8 B 1029/14, juris, Rdnr. 37.

Diesen Anforderungen genügt die erteilte Befreiung von den Verboten der Landschaftsschutzgebietsverordnung für das im Bauabschnitt 2 geplante Vorhaben. Die für die Reichweite der Genehmigung maßgeblichen Umstände ergeben sich aus dem angegriffenen Bescheid in Verbindung mit dem Landschaftspflegerischen Begleitplan vom 19.6.2019 und der Artenschutzprüfung Stufe II vom 19.6.2019.

Der angegriffene Bescheid bezeichnet das Vorhaben, für das die Befreiung erteilt wird, als ,Neubau und Modernisierung der Freianlagen auf der Insel Grafenwerth “ und verweist auf den gestellten Antrag. Damit gemeint ist der Landschaftspflegerische Begleitplan vom 19.6.2019, auf dessen Deckblatt sich ausdrücklich die Formulierung , , [...] und Antrag auf Befreiung von Ge- und Verboten des Landschaftsschutzes" findet. Auf Seite 3 wird nochmals ausdrücklich ausgeführt, dass ein Befreiungsantrag gestellt werde. Aus dem Bescheid ergibt sich (auch für einen ,unbeteiligten“ Dritten), dass der Antragsgegner den Landschaftspflegerischen Begleitplan als Antrag der Beigeladenen aufgefasst hat. Denn in dem Bescheid wird der Beigeladenen aufgegeben, die im Landschaftspflegerischen Begleitplan beschriebenen Vermeidungs-, Schutz- und Ausgleichsmaßnahmen vollständig umzusetzen. Dass die Beigeladene in ihrem Antrag vom 17.9.2019, den sie als ,formalen Antrag“ bezeichnete, nicht nochmals ausdrücklich auf den Landschaftspflegerischen Begleitplan Bezug nahm, führt wegen der ausdrücklichen Bezugnahme des Bescheids auf den Landschaftspflegerischen Begleitplan deshalb nicht zu Zweifeln am Antrags- und Regelungsinhalt. Teil der Antragsunterlagen ist zusätzlich auch die Artenschutzprüfung Stufe II, deren Inhalt ebenfalls zur Bestimmung der Reichweite des Genehmigungsinhalts heranzuziehen ist. Sie steht in einem untrennbaren Zusammenhang mit dem Landespflegerischen Begleitplan, was daraus ersichtlich ist, dass sie ebenfalls auf den 19.6.2019 datiert ist und im Verwaltungsvorgang des Antragsgegners dem Landschaftspflegerischen Begleitplan unmittelbar nachgeheftet ist, sogar noch vor dem „Bestandsund Konfliktplan“ und dem „Maßnahmenplan“, die ihrerseits noch Teil des Landschaftspflegerischen Begleitplans sind, wie sich aus dessen Inhaltsverzeichnis ergibt.

Den Antragsunterlagen ist hinreichend klar zu entnehmen, welche (Bau-)Maßnahmen im Bauabschnitt 2 an welchem Ort auf der Insel Grafenwerth durchgeführt werden sollen.

Auch bezogen auf die entlang der Promenade geplante Beleuchtung sind die Antragsunterlagen und damit der Genehmigungsinhalt hinreichend bestimmt. In der Artenschutzprüfung findet sich auf S. 22 eine bildliche Darstellung und textliche Erläuterung, wie die Beleuchtung erfolgen soll. Auch wenn das dort genannte „Beleuchtungskonzept" den Antragsunterlagen nicht beigefügt ist, ist noch hinreichend erkennbar, wie viele Leuchten an welchen Standorten aufgestellt werden sollen. Nicht mehr Gegenstand des Bescheids sind allerdings die Vorgaben für 
die Beleuchtung, die der Antragsgegner der Beigeladenen formlos per E-Mail vom 30.8.2019 (...) machte. Ob diese erforderlich sind, um die materiellen Voraussetzungen für eine Befreiung abzusichern, kann hier dahinstehen. Denn dies ist keine Frage der Bestimmtheit des Bescheids.

Der Bescheid ist auch nicht deshalb inhaltlich unbestimmt, weil er nicht die Verbote der Landschaftsschutzgebietsverordnung ausdrücklich benennt, von denen eine Befreiung erteilt wird. Im Landschaftspflegerischen Begleitplan und damit im Antrag der Beigeladenen werden die einschlägigen Verbote, von denen eine Befreiung beantragt wurde, ausdrücklich aufgeführt $(\$ 4$ Abs. $2 \mathrm{Nr} .1$ bis 4 , 13 und 16 LSGVO, vgl. S. 7 f. des Landschaftspflegerischen Begleitplans). Da der Antragsgegner dem Antrag der Beigeladenen vollständig entsprochen hat, lässt sich der Bescheid zudem nur so verstehen, dass er eine Befreiung von allen in der Landschaftsschutzgebietsverordnung (möglicherweise) entgegenstehenden $\mathrm{Ge}$ - und Verboten erteilen wollte.

Die Kammer lässt offen, ob der Bescheid auch bezogen auf den Bauabschnitt 3 hinreichend bestimmt ist. Hieran dürften aber Zweifel bestehen, weil sich der durch den Bescheid in Bezug genommenen Planung der Beigeladenen nicht hinreichend sicher entnehmen können lassen dürfte, welche baulichen Anlagen auf welcher Fläche errichtet werden. Im Landespflegerischen Begleitplan, S. 21, wird zwar erläutert, dass im Bauabschnitt 3 ein neuer Platzbereich erstellt werde, der den Mineralbrunnen im Zentrum, eine kleine asphaltierte Fläche mit Bühne und neue multifunktionale Spielflächen umfasse. Im Bestands- und Konfliktplan bzw. dem Maßnahmenplan finden sich dann auch mehrere versiegelte bzw. neu $\mathrm{zu}$ versiegelnde Flächen im Bauabschnitt 3. Unklar ist mangels Erläuterung aber, welche der Flächen welche Funktion haben soll. So ist etwa nicht zu erkennen, auf welcher der anzulegenden Flächen die Bühne (bzw. die Vorrichtungen für eine Bühne) errichtet werden soll(en). Dies wäre für die Bestimmtheit höchstens dann unbeachtlich, wenn der Antragsgegner der Beigeladenen die Wahl lassen wollte, auf welcher Fläche welche Anlage errichtet werden soll. Die Kammer geht im Folgenden davon aus, dass die Bühne auf der Fläche errichtet werden soll, die in dem von der Beigeladenen mit Schriftsatz vom 27. 4.2020 übersandten Plan (...) hierfür ausgewiesen ist. Gegenstand der Antragsunterlagen ist dieser Plan allerdings nicht. Auch die Verwaltungsvorgänge des Antragsgegners enthalten den Plan nicht.

2. Jedenfalls die tatbestandliehen Voraussetzungen der Befreiung liegen bezogen auf die Bauabschnitte 2 und 3 des Vorhabens nicht vor.

Grundlage der erteilten Befreiung ist $\$ 67$ Abs. 1 Satz 1 Nr. 1 BNatSchG. Hiernach kann u.a. von den Geboten und Verboten des Naturschutzes der Länder auf Antrag Befreiung gewährt werden, wenn dies aus Gründen des überwiegenden öffentlichen Interesses, einschließlich solcher sozialer und wirtschaftlicher Art, notwendig ist.

Die Erteilung einer Befreiung setzt eine atypische Sondersituation voraus, die der Verordnungsgeber beim Erlass der Verordnung nicht in den Blick genommen hat. Eine Befreiung kommt nur in Betracht, wenn die Anwendung der Geoder Verbotsnorm im Einzelfall zu einem Ergebnis führen würde, das dem Normzweck nicht mehr entspricht und deshalb normativ so nicht beabsichtigt ist. In derartigen (Sonder-)Fällen soll der generelle und damit zwangsläufig auch schematische Geltungsanspruch des betroffenen $\mathrm{Ge}$ - oder Verbots zugunsten der Einzelfallgerechtigkeit durchbrochen werden können, vgl. BVerwG, Urt. v. 26.3.1998 - 4 A 7.97, juris, Rdnr. 26; Beschl. v. 14.9.1992 - 7 B 130.92 -, juris, Rdnr. 5, und vom 20.2.2002 - 4 B 12.02, juris, Rdnr. 3 jeweils zu $\$ 31$ Abs. 1 BNatSchG a. F.); OVG Münster, Beschl. v. 30.1.2017 - 8 A 1205/14, juris, Rdnr. 9 ff.

Naturschutzrechtliche Befreiungen sind einzelfallbezogen und dienen nicht dazu, landschaftsrechtliche Regelungen in einem nicht unerheblichen Umfang außer Kraft $\mathrm{zu}$ setzen oder inhaltlich zu ändern. Sie sind nicht dafür konzipiert, bauliche Anlagen in nennenswertem Umfang in für den Landschaftsschutz bedeutsamen Teilen eines Landschaftsschutzgebiets oder gar flächendeckend zuzulassen und auf diese Weise einen allgemeinen, sich generell stellenden Konflikt zwischen Landschaftsschutz und anderen im öffentlichen Interessen liegenden Zielen aufzulösen, vgl. OVG Münster, Urt. v. 21.4.2020 - 8 A 311/19, juris, Rdnr. 70 f., m.w. N.; vgl. für $\$ 31$ Abs. 2 BauGB BVerwG, Beschl. v. 5.3.1999 - 4 B 5.99, juris, Rdnr. 5.

Eine Befreiung ist nicht erst dann notwendig, wenn dem öffentlichen Interesse auf keine andere Weise als durch die Befreiung entsprochen werden kann, sondern schon dann, wenn sie zur Erreichung der verfolgten Ziele vernünftigerweise geboten ist. Es genügt aber nicht, wenn die Befreiung dem allgemeinen Wohl nur irgendwie nützlich oder dienlich ist, vgl. BVerwG, Beschl. v. 5.2.2004 - 4 B 110.03, juris, Rdnr. 6 (zu \$31 Abs. 2 BauGB); OVG Münster, Beschl. v. 9.6.2017-8 B 1264/16, juris, Rdnr. 7.

Bestehen alternative Möglichkeiten, ohne unzumutbaren Aufwand das verfolgte Interesse zu verwirklichen (etwa Standortalternativen oder Ausführungsvarianten), die eine Befreiung nicht erfordern, kann deshalb in der Regel nicht davon ausgegangen, dass die Befreiung notwendig ist, vgl. OVG Münster, Urt. v. 11.9.2012 - 8 A 104/10, juris, Rdnr. 55; VGH Mannheim, Urt. v. 13.10.2005 - $3 \mathrm{~S}$ 2521/04, juris, Rdnr. 48.

Da die Gründe des öffentlichen Interesses überwiegen müssen, ist eine einzelfallbezogene bipolare Abwägung der durch die Befreiung verfolgten öffentlichen Interessen mit den durch die Landschafsschutzverordnung geschützten Interessen erforderlich. Eine „Saldierung“ aller für und gegen das Vorhaben sprechenden öffentlichen Interessen findet nicht statt. In die Abwägung sind deshalb nur das Gewicht der mit der Befreiung verfolgten öffentlichen Interessen, das Gewicht der durch die Landschaftsschutzverordnung geschützten Belange und das Ausmaß der durch das Vorhaben ausgelösten Beeinträchtigung einzustellen, vgl. BVerwG, Urt. v. 12.6.2019 - 9 A 2.18, juris, Rdnr. 127, und vom 9.7.2009 - 4 C 12.07, juris, Rdnr. 15 ff., und vom 12.3.2008 - 9 A 3.06, juris, Rdnr. 154, jeweils zu $₫ 34$ Abs. 3 Nr. 1 BNatSchG; OVG Münster, Beschl. v. 8.11.2017 - 8 A 2454/14, juris, Rdnr. 17.

Die im Rahmen des $₫ 67$ BNatSchG zu treffende Abwägung ist bereits Teil des Tatbestands der Norm und nicht erst im Rahmen einer Ermessensentscheidung zu berücksichtigen, vgl. BVerwG, Beschl. v. 20.2.2002 - 4 B 12.02, juris, Rdnr. 3 unter Verweis aufBVerwG, Urt. v. 26.3.1998 - 4 A 7.97, juris (zu der $\$ 67$ BNatSchG vergleichbaren Vorschrift des $₫ 31$ Abs. 1 Satz 1 Nr. 2 BNatSchG a.F.); OVG Münster, Urt. v. 11.9.2012 - 8 A 104/10, juris, Rdnr. 42.

Gründe dafür, die gerichtliche Kontrolldichte bei der Überprüfung dieses Tatbestandsmerkmals einzuschränken, bestehen nicht. Die Abwägung im Rahmen der Erteilung einer naturschutzrechtlichen Befreiung ist nicht durch planerische Gestaltungsfreiheit geprägt, mithin gerichtlich voll überprüfbar. Das Gericht muss deshalb ggf. selbst feststellen, ob die die Befreiung tragenden öffentlichen Interessen überwiegen, ob also das Tatbestandsmerkmal vorliegt, vgl. VG Aachen, Urt. v. 7.5.2012 - 6 K 1140/10 -, juris, Rdnr. 119; Gellermann, in: Landmann/Rohmer, Umweltrecht, Stand: 91. EL September 2019, \$67 BNatschG, Rdnr. 12; Lau, in: Frenz/Müggenborg, BNatSchG, 2. Aufl. 2016, $\$ 67$ Rdnr. 6 (zu $\$ 67$ Abs. 1 Satz 1 Nr. 2 BNatSchG); a.A.: OVG Berlin, Urt. v. 28.9.2012 - 11 S 61.12, juris, Rdnr. 5; VGH Mannheim, Urt. v. 14.3.2011 - 5 S 644/09, juris, Rdnr. 48 (zum dortigen Landesrecht).

Ausgehend hiervon liegen die tatbestandliehen Voraussetzungen für die erteilte Befreiung bezogen auf den Bauabschnitt 2 und 3 nicht vor. Zwar ist wegen des vom Normgeber nicht vorhergesehenen besonders hohen Besucherdrucks auf die Insel Grafenwerth eine atypische Sondersituation anzunehmen (unten a). Das Vorhaben verfolgt auch im öffentlichen Interesse liegende Ziele, indem es zum einen Besucher von für 
schützenswerter erachteten Teilen der Insel fernhalten (Besucherlenkung) und zum anderen die Erholungsmöglichkeiten auf der Insel steigern soll (unten b). Die im Bauabschnitt 2 geplante Sitzstufentreppe am Rhein ist zur Besucherlenkung aber schon nicht geeignet und damit nicht notwendig. Die Steigerung der Erholungsmöglichkeiten allein vermag den Bau der Sitzstufentreppe nicht zu rechtfertigen, weil dieses Interesse nicht die durch die Landschaftsschutzgebietsverordnung geschützten Belange überwiegt (unten c). Die Befreiung für eine im Bauabschnitt 3 geplante Fläche für eine Bühne ist schon deshalb rechtswidrig, weil hierdurch das in der Landschaftsschutzgebietsverordnung vorhergesehene Verbot für Veranstaltungen auf der Insel Grafenwerth faktisch funktionslos würde (unten d). Auch wenn einzelne der in den Bauabschnitten 2 und 3 geplanten Anlagen voraussichtlich genehmigungsfähig sein dürften, rechtfertigt dies nicht, die Wiederherstellung der aufschiebenden Wirkung auf die Sitzstufentreppe bzw. die Bühne zu begrenzen (unten e).

a) Die eine Befreiung grundsätzlich ermöglichende atypische Situation liegt vor, weil die Insel Grafenwerth einem besonders hohen Besucherdruck ausgesetzt ist.

Die Einschätzung von Beigeladener und Antragsgegner, die Insel Grafenwerth sei einem besonders hohen Besucherdruck ausgesetzt, ist in tatsächlicher Hinsicht vor dem Hintergrund der Lage der Insel Grafenwerth, die das Rheinufer für die Stadt Bad Honnef ersetzt, plausibel. Der Landungssteg der Köln Düsseldorfer/Deutsche Rheinschifffahrt $\mathrm{GmbH}$, ein Ausflugrestaurant, ein Freibad und neun Tennisplätze belegen die touristische Erschließung und das Interesse an der Insel. Ein Indiz für den hohen Besucherdruck findet sich auch in der Artenschutzprüfung Stufe II, in der auf S. 10 ausgeführt wird, dass der Einsatz von Horchkisten bei der Fledermauserfassung wegen des starken auch nächtlichen Besucherdrucks habe eingeschränkt werden müssen. Die hohe Besucherzahl wird letztlich auch vom Antragsteller nicht durchgreifend in Zweifel gezogen, nachdem dieser zwar bemängelt, dass die unterschiedlichen Besuchergruppen und deren typisches Verhalten auf der Insel von Antragsgegner und Beigeladenen nicht erfasst worden seien. Die hohe Anzahl von Besuchern auf der Insel hat er hingegen nicht substantiiert in Frage gestellt.

Baumaßnahmen, die den Verboten der Landschaftsschutzgebietsverordnung widersprechen, als (notwendige) Reaktion auf eine besonders hohe Besucherzahl wurden vom Normgeber nicht vorhergesehen. Dies lässt sich schon aus der Verordnung selbst ableiten, weil der Normgeber ausweislich von $\$ 4$ Abs. 2 Nr. 1.1. vierter Spiegelstrich LSGVO davon ausgegangen ist, eine Besucherlenkung könne ausreichend durch eine Beschilderung erfolgen. Nach dieser Vorschrift ist es verboten, bauliche Anlagen zu errichten, zu ändern oder in ihrer Nutzung zu ändern. Ausgenommen hiervon sind (nur) Schilder, die auf die Schutzausweisung hinweisen oder der Besucherlenkung und -information des Schutzgebietes dienen. Dass eine Situation eintreten könnte, in der Schilder zur Besucherlenkung wegen einer besonders hohen Besucherzahl nicht mehr ausreichend sind, hat der Normgeber also nicht vorhergesehen.

Unabhängig hiervon handelt es sich bei dem Vorhaben der Beigeladenen auch um ein singuläres Ereignis, das schon deshalb von dem Normgeber bei der Schaffung der Verbotsnormen nicht in den Blick genommen werden konnte. Es würde zu kurz greifen, die Planung der Beigeladenen nur als die Errichtung mehrerer baulicher Anlagen und Wege zu begreifen, die nach der Landschaftsschutzgebietsverordnung gerade ausdrücklich verboten sind ( $\$ 4$ Abs. 2 Nr. 2 LSGVO). Vielmehr ist die Planung einheitlich zu betrachten und stellt sich danach als eine weitgehende Umgestaltung der Erholungs- bzw. Freizeitmöglichkeiten auf der Insel Grafenwerth dar, durch die diese Nutzungen der Insel erstmals räumlich klar von dem von Beigeladener und Antragsgegner für schützenswert gehaltenen Südostteil der Insel abgegrenzt werden sollen, vgl. zur Begründung einer atypischen Sondersitua- tion wegen der Singularität eines Vorhabens BVerwG, Beschl. v. 12.4.2005 - 9 VR 41.04, juris, Rdnr. 36 und Urt. v. 26.3. 1998 - 4 A 7.97, juris, Rdnr. 26.

b) Die mit dem Vorhaben verfolgte Ziele liegen im öffentlichen Interesse.

Die Beigeladene möchte mit den geplanten baulichen Anlagen eine besondere Anreizwirkung auf die Besucher der Insel auslösen, um diese hierdurch vom Ostufer am Altarm des Rheins und von der naturnahen Südspitze fernzuhalten. Diese Bereiche sollen dem Naturschutz vorbehalten bleiben. Der Schutz von Teilen des Ostufers und der Südspitze liegt im öffentlichen Interesse, weil diese Teile der Insel nicht nur (wie der Rest der Insel) als Landschaftsschutzgebiet und als Teil der Biotopverbundfläche von besonderer Bedeutung ,Rhein zwischen Landesgrenze und Römlinghoven“, sondern zusätzlich noch als Biotopverbundfläche mit herausragender Bedeutung „Rheinufer mit Weichholzauenresten auf der Insel Grafenwerth " geschützt sind. Der Gehölzbestand am Ostufer ist zudem Teil des Biotopkatasters ,Rheinufer am Grafenwerth“.

Daneben verfolgt das Vorhaben zusätzlich das Ziel, die Möglichkeiten zur Erholung bzw. Freizeitgestaltung auf der Insel Grafenwerth für die Besucher zu verbessern.

Auch dieses Ziel liegt als Teil der öffentlichen Daseinsfürsorge im öffentlichen Interesse.

c) Die im Bauabschnitt 2 geplante Sitzstufenanlage ist zur Besucherlenkung nicht notwendig, weil sie dieses Ziels nicht fördert.

Die Sitzstufenanlage aus Betonfertigteilen am Einmündungsbereich zum Freibadzugang ist das zentrale Element des Bauabschnitts 2. Sie soll nach den Angaben der Beigeladenen den Endpunkt der Promenade markieren. Diese Aussage insinuiert, die Promenade ende baulich an dieser Stelle, sodass Besucher von einem weiteren Gang in Richtung Süden (und damit in als schützenswerter erachtete Teile) der Insel abgehalten werden. Dies trifft allerdings in tatsächlicher Hinsicht nicht zu. Auf Satellitenbildern der Internetseite GoogleMaps und auf den von der Beigeladenen übersandten Fotos ist deutlich zu erkennen, dass ein asphaltierter Weg auch nach dem geplanten Standort der Sitzstufenanlage weiter nach Süden verläuft, erst parallel zum R hein und dann südlich entlang der Tennisplätze. Ein nichtasphaltierter Weg (Trampelpfad) verlängert den asphaltierten Weg dann sogar bis zur Südspitze der Insel. Von dort verläuft dann ein weiterer Trampelpfad entlang des Ostufers der Insel wieder nach Norden. Das durch den streitgegenständlichen Bescheid zugelassene Vorhaben beinhaltet nicht, dass der nach Süden führende Weg verändert, zurückgebaut oder baulich blockiert wird. Auch wenn die Sitzstufenanlage wegen ihrer Nähe zum R hein Besucher zum Aufenthalt einladen und anlocken dürfte, lässt dies deshalb nicht den Schluss zu, dass diese (wegen des weiter vorhandenen Weges) nicht von dort aus den Süden der Insel erkunden werden. Die Anlage der Treppe an einem Weg, der in die schützenswerten Teile der Insel führt, hat möglicherweise sogar den genau entgegengesetzten Effekt, nämlich mehr Besucher nach einen „Zwischenstopp" in diese Bereiche zu führen.

Inwieweit die Sitzstufenanlage für das mit dem Vorhaben ebenfalls verfolgte Ziel, die Erholungsmöglichkeiten zu verbessern, notwendig ist, kann dahinstehen. Denn jedenfalls überwiegt dieses Interesse für sich allein nicht die durch die Landschaftsschutzgebietsverordnung geschützten Belange. Die Erholungsmöglichkeiten auf der Insel würden durch die Sitzstufenanlage im Vergleich zum bereits bestehenden $\mathrm{Zu}$ stand nur in einem geringen Maße gefördert. Zwar würde die Sitzstufenanlage den Besuchern der Insel die Möglichkeit eröffnen, sich in der Nähe des Rheins aufzuhalten, dort zu rasten und dabei die Landschaft (insbesondere den Rhein) zu betrachten. Es existieren aber bereits andere Aussichtspunkte und Rastmöglichkeiten auf der Insel, auch die Promenade bietet einen freien Blick auf den Rhein. Erkauft würden die Vorteile der Sitzstufenanlage durch einen durch die bauliche Verände- 
rung begründeten nicht unerheblichen Eingriff in die Uferböschung. Noch schwerer ins Gewicht fallen aber die Schäden, die von den Besuchern am Rheinufer zu befürchten wären, die durch die Anlage gezielt in die Nähe des Rheins gelenkt würden, der in diesem Bereich - so wie auch die Uferböschung selbst - zum FFH-Gebiet „Rhein Fischschutzzonen zwischen Emmerich und Bad Honnef" gehört. In der von der Beigeladenen vorgelegten „FFH-Verträglichkeitsprüfung“ (...) wird dazu ausgeführt, eine Erhöhung des Besucheraufkommens am Rhein durch den Bau der Sitzstufenanlage sei zu vermeiden, weil die Gefahr bestehe, dass benachbarte empfindliche Uferabschnitte an der Südspitze der Insel durch Spaziergänger (mit möglicherweise Grillen und Vermüllung) und Hunde aufgesucht würden. Auf dem verbleibenden unteren Teil der Uferböschung seien die Blocksteine zu erhalten. Diese Steinpackung halte aufgrund der lockeren Schichtung das Betreten der Ufer weitgehend ab. Des Weiteren sei durch eine Informationstafel auf die Bedeutung und Empfindlichkeit der Uferzone hinzuweisen. Die Beigeladene geht demnach selbst davon aus, dass der Bereich, in den die Sitzstufenanlage gebaut wird, schützenswert und empfindlich ist und Besucher in schützenswerte Teile lenken könnte. Auch wenn sie augenscheinlich mögliche Schäden durch Benutzer der Sitzstufenanlage vorzubeugen versucht, entsteht zumindest ein erhöhtes und nicht unerhebliches Risiko für durch Besucher verursachte Schäden. Diese zu befürchtenden Schäden würden das Schutzziel in $\$ 3$ Abs. 2 lit. a) LSGVO erheblich beeinträchtigen, wonach die Unterschutzstellung des Landschaftsschutzgebiets zur Erhaltung, Entwicklung und Wiederherstellung der Leistungs- und Funktionsfähigkeit des Naturhaushalts sowie der Regenerationsfähigkeit und nachhaltigen Nutzungsfähigkeit erfolgt. Selbst wenn das durch die Sitzstufenanlage geförderte Ziel der Erholung ebenfalls als Ziel der Unterschutzstellung ( $\$ 3$ Abs. 2 lit. c) LSGVO) eingeordnet würde, wären die nur gering zu bewertenden Vorteile für die Erholungsnutzung der Insel nicht höher zu bewerten als die durch die Sitzstufenanlage hervorgerufenen Gefahren für den durch $₫ 3$ Abs. 2 lit. a) LSGVO geschützten Belang.

Liegen schon keine überwiegenden Interessen vor, die die Befreiung für den Bau der Sitzstufentreppe rechtfertigten können, kann dahinstehen, ob der Befreiung auch entgegensteht, dass die Durchführung des Bauvorhaben ohnehin nicht möglich sein könnte, weil eine FFH-Verträglichkeitsprüfung nach $₫ 34$ BNatschG bislang im Rechtssinne nicht erfolgt ist. Gemäß \$34 Abs. 1 Satz 1 BNatSchG sind Projekte vor ihrer Zulassung oder Durchführung auf ihre Verträglichkeit mit den Erhaltungszielen eines Natura 2000-Gebiets zu überprüfen, wenn sie einzeln oder im Zusammenwirken mit anderen Projekten oder Plänen geeignet sind, das Gebiet erheblich $\mathrm{zu}$ beeinträchtigen, und nicht unmittelbar der Verwaltung des Gebiets dienen. Der Antragsgegner hat im Gerichtsverfahren ausdrücklich erklärt, eine Verträglichkeitsprüfung sei nicht Gegenstand des Bescheids, weil diese nur im Falle einer (hier nicht erteilten) Befreiung nach $\$ 67$ Abs. 1 Satz 1 Nr. 2 BNatSchG durchzuführen sei. Er (als zuständige Behörde) hat die Prüfung demnach jedenfalls nicht durchgeführt, auch wenn die Beigeladene Unterlagen für eine solche Prüfung in Erfüllung der Pflicht aus $₫ 34$ Abs. 1 Satz 3 BNatSchG vorgelegt hatte. Die dieser Erklärung immanente Vorstellung, im Fall einer Befreiung nach $\$ 67$ Abs. 1 Nr. 1 $\mathrm{BNatSchG}$ sei eine Verträglichkeitsprüfung nie durchzuführen, dürfte nicht zu halten sein. Die Pflicht zur Verträglichkeitsprüfung ist unionsrechtlich vorgegeben und knüpft an den Begriff des Projekts an. Sie ist ein Verfahrensschritt innerhalb des die Zulassung eines Projekts betreffenden behördlichen Entscheidungsprozesses. Auf welcher Grundlage die Zulassung des Projekts erfolgt ist für das Erfordernis einer Verträglichkeitsprüfung nicht von Belang, vgl. BVerwG, Urt. v. 10.4.2013 - 4 C 3.12, juris, Rdnr. 11; vgl. auch OVG Münster, Urt. v. 11.9.2012 - 8 A 104/10, juris, Rdnr. 32 ff.

Dahinstehen kann, ob der Antragsgegner die Durchführung der Verträglichkeitsprüfung auf die Bezirksregierung verlagern durfte, die am 17.1.2020 eine wasserrechtliche Erlaubnis für die Sitzstufenanlage erteilt hat, was denkbar ist. Denn auch die Bezirksregierung hat eine Verträglichkeitsprüfung im Rechtssinne nicht durchgeführt. Die Bezirksregierung hat sich ausweislich des im Verwaltungsvorgangs vorhandenen E-Mail-Austausches zwar offenbar das Ergebnis der von der Beigeladenen vorgelegten „Verträglichkeitsprüfung" zu eigen machen wollen. Gegenstand der wasserrechtlichen Erlaubnis ist diese Prüfung aber nicht geworden. Die wasserrechtliche Erlaubnis listet auf Seite 1 auf, welche Unterlagen geprüft und Bestandteil der Genehmigung seien. Die Unterlagen der Beigeladenen zur Verträglichkeitsprüfung werden dabei nicht genannt. Auch ansonsten finden sich in der wasserrechtlichen Erlaubnis keine Hinweise auf die Durchführung einer Verträglichkeitsprüfung.

d) Die Erteilung der Befreiung für die im Bauabschnitt 3 für eine Bühne für verschiedenste Veranstaltungen vorgesehene asphaltierte Fläche ist schon deshalb rechtswidrig, weil sie bei Betrachtung ihrer Folgen nicht mehr als einzelfallbezogene Regelung erweist, sondern vielmehr die Verbotsvorschrift des $\int 4$ Abs. 2 Nr. 7 LSGVO funktionslos machen würde. Nach $\$ 4$ Abs. 2 Nr. 7 LSGVO ist es verboten, Veranstaltungen mit mehr als 100 Personen außerhalb der befestigten Wege [...] oder den dafür vorgesehenen Flächen abzuhalten. Auf der Insel Grafenwerth ist eine Fläche in der geplanten Größe, die gezielt für solche Veranstaltungen vorgesehen ist und die für eine Bühne notwendige Infrastruktur vorhält, bislang nicht vorhanden.

Die Verbotsvorschrift hat auf Grund der derzeitigen Bebauung der Insel zur Folge, dass große Veranstaltungen auf der Insel grundsätzlich verboten sind, weil es an einer dafür nutzbaren Fläche fehlt. Sie können deshalb nur im Einzelfall unter Erteilung einer Ausnahme nach $\$ 7$ Abs. 2 LSGVO zugelassen werden. Nach dieser Vorschrift kann der Antragsgegner von den Verboten des $\$ 4$ Abs. 2 LSGVO auf Antrag auf Grund eines mit dem Antragsgegner abgestimmten Konzeptes eine Ausnahme erteilen, wenn diese dem besonderen Schutzzweck des $\$ 3$ LSGVO nicht zuwiderläuft und den Charakter des Landschaftsschutzgebietes nicht verändert. Alternativ kommt die Zulassung einer Veranstaltung nur unter den noch strengeren Voraussetzungen einer naturschutzrechtlichen Befreiung in Betracht. Der Antragsgegner hatte Ausnahmen für solche Veranstaltungen in der Vergangenheit mehrmals erteilt. Im Bauabschnitt 3 würde nun erstmals eine große Fläche geschaffen, auf der (große) Veranstaltungen in Zukunft ohne Verstoß gegen die Verbotsvorschrift (und damit ohne das Erfordernis einer Ausnahme oder einer naturschutzrechtlichen Befreiung für jede einzelne Veranstaltung) durchgeführt werden könnten. Damit würde der Charakter des Schutzgebietes aber wesentlich verändert. Die faktische Aufhebung einer Verbotsvorschrift und die durch sie ausgelöste Veränderung in der Nutzung der Insel ist einer Änderung der Verordnung vorbehalten und darf nicht durch eine naturschutzrechtliche Befreiung erfolgen. Der Antragsgegner darf nicht durch Verwaltungsakt wesentliche Inhalte der Rechtsverordnung faktisch auBer Kraft setzen, vgl. BVerwG, Beschl. v. 5.3.1999 - 4 B 5.99, juris, Rdnr. 5 f., m.w. N. (zu $\$ 31$ Abs. 2 BauGB).

e) Nachdem die Befreiung hinsichtlich der Sitzstufenanlage und der für eine Bühne vorgesehenen Fläche rechtswidrig ist, kann der Bescheid nicht für die übrigen Maßnahmen in den Bauabschnitten 2 und 3 teilweise aufrechterhalten werden. Es fehlt an der hierfür notwendigen Teilbarkeit des Verwaltungsaktes.

Ein angefochtener Verwaltungsakt ist teilbar, wenn die rechtlich unbedenklichen Teile nicht in einem untrennbaren inneren Zusammenhang mit dem rechtswidrigen Teil stehen, vgl. BVerwG, Urt. v. 13.11.1997 - 3 C 33.96, juris, Rdnr. 23; und Beschl. v. 2.5.2005 - 6 B 6.05, juris, Rdnr. 8.

Im Fachplanungsrecht ist eine räumliche Teilbarkeit eines Vorhabens anerkannt, wenn der Verwaltungsakt auch ohne den von dem Rechtsmangel erfassten Regelungsteil 
eine selbständige und rechtmäßige, vom Träger des Vorhabens sowie von der Planungsbehörde auch so gewollte Planung zum Inhalt hat. Dem Planungsträger darf nicht ein (Rest-)Vorhaben aufdrängt werden, das er in dieser Gestalt gar nicht verwirklichen möchte, vgl. BVerwG, Urt. v. 21.2.1992 - 7 C 11.91, juris, Rdnr. 23; und Beschl. v. 7.12.1988 - 7 B 98.88, juris, Rdnr. 9.

Dieser Maßstab lässt sich auch außerhalb des Fachplanungsrechts zumindest dann anlegen, wenn -so wie hier - ein Vorhaben sich auf einen größeren räumlichen Bereich bezieht und sich aus einer Vielzahl von Einzelmaßnahmen (baulichen Anlagen) zusammensetzt. Bereits die von der Beigeladenen vorgenommene Unterteilung des Vorhabens in drei Bauabschnitte spricht dafür, diese nach ihrem Verständnis jeweils in sich als Einheit anzusehen sind. Vor allem aber sind sowohl die Sitzstufentreppe als auch die Fläche für eine Bühne jeweils das wesentliche Element der beiden Bauabschnitte. Die weiteren Maßnahmen in den beiden Bauabschnitten sind zumindest teilweise auf sie bezogen, etwa die geplanten Änderungen der Wegführung im Bauabschnitt 3 oder auch die Veränderung der zu der Sitzstufenanlage führenden Promenade, setzen also deren Verwirklichung voraus. Diese weiteren Maßnahmen können die ihnen zugeschriebenen Funktionen in der Planung ohne den Bau der Bühnenfläche und der Sitzstufenanlage nicht mehr übernehmen. Der Bescheid kann deshalb nicht teilweise aufrechterhalten werden, ohne der Beigeladenen eine von ihr so nicht gewollte und von dem Antragsgegner nicht geprüfte und erlaubte Planung aufzudrängen.

3. Der Verstoß gegen die Voraussetzungen des $\$ 67$ Abs. 1 BNatSchG berührt Belange des Natur- und Landschaftsschutzes. Diese gehören zu den Zielen, die der Antragsteller nach seiner Satzung (vgl. deren $\$ 1$ Abs. 2, Abs. 3) fördert. Er kann den Verstoß deshalb rügen, $\$ 2$ Abs. 4 Satz 1 UmwRG.

$\mathrm{Ob}$ der Verstoß im Hauptsacheverfahren zur Aufhebung der Befreiung oder nur zur Feststellung deren Rechtswidrigkeit führen wird, kann dahinstehen. Nach $\$ 7$ Abs. 5 Satz 1 UmwRG führt eine Verletzung materieller Rechtsvorschriften nur dann zur Aufhebung der Entscheidung, wenn sie nicht durch Entscheidungsergänzung oder ein ergänzendes Verfahren behoben werden kann. Die Vorschrift ist orientiert an der Regelung der Planerhaltung nach $\$ 75$ Abs. 1a VwVfG, vgl. BT-Drs. 18/9526, S. 44f.

Im Fall einer möglichen Entscheidungsergänzung im Planfeststellungsverfahren geht das Bundesverwaltungsgericht zwar davon aus, dass ein Antrag auf vorläufigen Rechtsschutz abzulehnen ist, vgl. BVerwG, Beschl. v. 19. 5. 2005 - 4 VR 2000.05, juris, Rdnr. 35.

Lassen sich die Fehler hingegen höchstens in einem ergänzenden Verfahren beheben, rechtfertigt bereits dies im Verfahren auf vorläufigen Rechtsschutz in der Regel die Aussetzung der Vollziehung, vgl. Seibert, NVwZ 2018, 97, 103.

Eine Entscheidungsergänzung liegt nur dann vor, wenn die ansonsten unveränderte Entscheidung um zusätzliche Regelungen ergänzt werden kann, um den Fehler zu beheben. Hier ist aber nicht ersichtlich, um welche zusätzlichen Regelungen der Bescheid ergänzt werden sollte, um die beschriebenen Verstöße zu beheben. Wenn die Verstöße überhaupt behebbar sein sollten, dürfte es dafür einer grundlegend überarbeiteten Planung bedürfen.

4. Der Antrag hat auch hinsichtlich der Eingriffsgenehmigung Erfolg. Dieser fehlt die Grundlage, nachdem sich die Befreiung als rechtswidrig erweist, weil die Eingriffsgenehmigung akzessorisch zur naturschutzrechtlichen Befreiung ist, vgl. $\$ 17$ Abs. $1 \mathrm{BNatSchG.}$

II. Der Antrag ist unbegründet, soweit der angegriffene Bescheid das Vorhaben in Bauabschnitt 1 betrifft.

Der Antragsgegner hat die Anordnung der sofortigen Vollziehung dem Erfordernis des $\$ 80$ Abs. 3 Satz 1 VwGO entsprechend begründet. Die Interessenabwägung zwischen dem Interesse an der sofortigen Vollziehung des Bescheids und dem Aussetzungsinteresse des Antragstellers fällt zu dessen Lasten aus. Der angegriffene Bescheid ist bezogen auf den
Bauabschnitt 1 mit überwiegender Wahrscheinlichkeit rechtmäßig (unten 1 .). Es liegen auch Gründe vor, aus denen die Anordnung des Sofortvollzugs gerechtfertigt ist (unten 2.).

1. Der angegriffene Bescheid ist hinreichend bestimmt (unten a). Auch die tatbestandliehen Voraussetzungen für die erteilte Befreiung liegen vor. Es liegt eine die Befreiung ermöglichende atypische Situation vor und das Vorhaben verfolgt im öffentlichen Interesse liegende Ziele (unten b). Das Vorhaben ist für die Erreichung dieser Ziele notwendig (unten c) und diese Ziele überwiegen auch die durch die Landschaftsschutzgebietsverordnung geschützten Belange (unten d). Ermessensfehler bei der Erteilung der Befreiung sind nicht ersichtlich. Der Bescheid kann für den Bauabschnitt 1 teilweise aufrechterhalten werden (unten e). Auch die Eingriffsgenehmigung ist rechtmäßig (unten f).

a) Der angegriffene Bescheid genügt den Bestimmtheitsanforderungen des $\$ 37$ Abs. 1 VwVfG NRW. Sein Regelungsgehalt, also die Reichweite der erteilten Befreiung, ist bestimmbar auf Grundlage des Antrags der Beigeladenen und der dazu von ihr überreichten Unterlagen (oben I. 1.).

Dass die Angaben zu den Ausgleichsmaßnahmen M3 bis M6 (Anlegung von Rasenflächen und Zierpflanzenrabatten, Ansaat eines blütenreichen Schattensaums, Anlage von extensiven Wiesenflächen, Entsiegelung und Anlage von Wiesenflächen, vgl. S. $27 \mathrm{f}$. des Landespflegerischen Begleitplans) keine Vorgaben über die Aussaat im Einzelnen zu nutzenden Arten enthalten, führt nicht auf die Unbestimmtheit des Bescheids. Die Angaben in dem Antrag (und damit auch der Inhalt der Befreiung) lassen ausreichend erkennen, welchen Charakter die betroffenen Flächen haben sollen. Der Bescheid überlässt der Beigeladenen mangels einer weiteren Eingrenzung demnach zwar einen gewissen Spielraum, wie die Maßnahmen konkret umgesetzt werden, ohne ihr aber wegen des beschriebenen Ziels der Maßnahmen einen völligen Freiraum zu lassen.

b) Die eine Befreiung eröffnende atypische Situation liegt vor, weil die Insel Grafenwerth einem besonders hohen Besucherdruck ausgesetzt ist (oben I. 2. a). Das Vorhaben verfolgt auch im öffentlichen Interesse liegende Ziele, indem es zum einen Besucher von für schützenswerter erachteten Teilen der Insel fernhalten (Besucherlenkung) und zum anderen die Erholungsmöglichkeiten auf der Insel steigern soll (oben 1. 2. b).

c) Das Vorhaben in Bauabschnitt 1 ist zur Erreichung dieser Ziele auch notwendig.

Die im Bauabschnitt 1 geplanten Wege sind geeignet, Besucher von dem Südostteil der Insel wegzuleiten, fördern dieses Ziel also. Durch den Bau einer neuen Treppenanlage werden Besucher von der nördlichen auf die Insel führenden Brücke auf den Rundweg um die Nordspitze der Insel geleitet und damit vom Süden der Insel weggelenkt. Der Einwand des Antragstellers hiergegen, der Bau der Treppe leite die Besucher gerade erst auf den Ostuferweg, vermag diese Einschätzung nicht in Zweifel zu ziehen. Zwar trifft es zu, dass durch den Bau der Treppe auch der Ostuferweg leichter als bislang erreichbar ist. Da die in den Norden der Insel führenden Wege aber attraktiver (mit Ziergesträuch und Zierpflanzenrabatten) und vor allem besser begehbar ausgestaltet werden als der Ostuferweg und zudem die Spielinseln im Norden (und nicht an dem nach Süden führenden Ostuferweg) angesiedelt werden, ist die der Planung immanente Vorstellung plausibel, die Besucher werden sich von der Treppe nach Norden (und nicht nach Süden) wenden. Von der Nordspitze, mit Blick rheinabwärts auf den Drachenfels, gelangen die Besucher zur Promenade am Westufer der Insel, von der aus sie einen freien Blick auf den Rhein haben. Es ist auch anzunehmen, dass die vier neuen Spiel-Inseln für unterschiedliche Altersklassen zumindest auf einen Teil der Besucher, vor allem auf Familien mit Kindern, eine besondere Anziehungskraft haben können und dadurch einen Aufenthalt dieser Nutzergruppe im Südostteil der Insel zusätzlich unwahrscheinlicher machen. 
Auch die Möglichkeiten, die Insel Grafenwerth für die Erholung zu nutzen, werden durch die im Bauabschnitt 1 geplanten Maßnahmen verbessert. Die Erneuerung bzw. teilweise auch erstmalige Anlage von barrierefreien Wegen macht die Nordspitze der Insel besser zugänglich und ermöglicht, zumindest erleichtert auch in ihrer Mobilität eingeschränkten Personen den Besuch und die Teilhabe an diesem Landschaftserleben. Sie können diesen Bereich der Insel erstmals visuell erfassen und sich dort aufhalten. Der bislang auf der Insel vorhandene Spielplatz ist nach der unwidersprochenen Darstellung von Antragsgegner und Beigeladener veraltet und insbesondere überlastet. Die neuen Spielinseln bieten für deutlich mehr Kinder bzw. Jugendliche und dies getrennt nach verschiedenen Altersgruppen Spielmöglichkeiten.

Alternativstandorte für die Spielinseln drängen sich nicht auf. Die Beigeladene hat nachvollziehbar erläutert, dass die für die Ausgleichsmaßnahme vorgesehene Wiese als Standort schlechter geeignet wäre. Der im Bauabschnitt 1 vorgesehene Standort befindet sich näher an der Gastronomie, sodass Eltern von dort in Sichtweite der Spielflächen (zumindest der Kleinkinder-Spielfläche) bleiben können. Dies rechtfertigt die Annahme, dass die Spielflächen dort besser angenommen werden als auf der Wiese. Diese ist im Übrigen wegen eines geringen Baumbestands in weiten Teilen nicht beschattet, sodass auch deshalb die Spielflächen insbesondere bei starker Sonneneinstrahlung wenig attraktiv und möglicherweise sogar gesundheitsgefährdend wären. Schließlich hat die Beigeladene zu Recht darauf hingewiesen, dass die Spielflächen auf der Wiese näher an dem südöstlichen Teil der Insel positioniert wären, also Besucher näher zu dem Teil der Insel führen würden, von dem sie gerade weggeleitet werden sollen. Andere Flächen (z.B. Flächen in der Mitte der Insel) drängen sich als gleich geeignete Alternativstandorte ebenfalls nicht auf. Auch sie wären nicht in der Nähe der (Außen-)Gastronomie angesiedelt bzw. würden Besucher näher zum südöstlichen Teil der Insel leiten.

d) Die mit dem Bauabschnitt 1 verfolgten Ziele, den südöstlichen Teil der Insel zu schützen und die Möglichkeiten zur Erholung auf der Insel zu verbessern, überwiegen auch die von der Landschaftsschutzgebietsverordnung geschützten Belange.

Die Rüge des Antragstellers, der Antragsgegner habe schon die in die Abwägung einzustellenden Belange nicht ausreichend ermittelt, dürfte zwar in tatsächlicher Hinsicht zutreffen, führt aber nicht zur Rechtswidrigkeit des Bescheids. Die Abwägung ist, wie ausgeführt, Teil des Tatbestands der Norm und gerichtlich voll überprüfbar. Allein entscheidend ist deshalb, ob in der Sache die mit dem Vorhaben verfolgten Ziele die durch die Landschaftsschutzgebietsverordnung geschützten Belange überwiegen. Dies ist der Fall.

Der im Bauabschnitt 1 ausgelöste Eingriff ist zwar gewichtig, nicht aber als besonders schwer einzuordnen.

Der Eingriff steht in Konflikt mit den in $\$ 3$ Abs. 2 lit. a) und lit. b) LSGVO genannten Zielen. Nach diesen Vorschriften erfolgt die Unterschutzstellung zur Erhaltung, Entwicklung und Wiederherstellung der Leistungs- und Funktionsfähigkeit des Naturhaushalts sowie der Regenerationsfähigkeit und nachhaltigen Nutzungsfähigkeit der Naturgüter und wegen der Vielfalt, Eigenart und Schönheit des Landschaftsbildes und der besonderen kulturhistorischen Bedeutung der Landschaft. Die Baumaßnahmen greifen in den Naturhaushalt ein und verändern auch das Landschaftsbild.

Aus der während der Umsetzung des Bauabschnitts 1 in Anspruch genommenen Fläche, die nach den Angaben zur Eingriffsregelung (S. 29 des Landespflegerischen Begleitplans) $4498 \mathrm{~m}^{2}$ beträgt, lässt sich ableiten, dass es sich nicht nur um eine ,punktuelle“ (zu vernachlässigende) Maßnahme, sondern ein größeres Vorhaben handelt. Es finden gegenüber dem bisherigen Zustand wesentliche Veränderungen statt, indem bisher unversiegelte Flächen erstmals versiegelt werden und bauliche Anlagen in Form von Spielgeräten erstmals errichtet werden. Dadurch wird der bis- herige Charakter der Nordspitze verändert, der im Landespflegerischen Begleitplan als ,naturnah ausgeprägt“ (S. 4) bezeichnet wird. Die Beigeladene gibt im Rahmen der Eingriffsregelung auch selbst an, dass nach der von ihr angelegten Bewertungsmethode nach dem Eingriff der Bereich des Bauabschnitts 1 zwei Drittel der ermittelten Biotopwertpunkte verliert. Der innere Bereich des Bauabschnitts 1 wird im Ergebnis erstmalig als durch menschliche Einflüsse gezielt beeinflusstes Gebiet erscheinen.

Gemindert wird die Schwere des Eingriffs allerdings dadurch, dass es sich schon jetzt nicht um ein vollkommen unberührtes Gebiet handelt. Der Rundweg um die Nordspitze war bereits zuvor existent und wurde genutzt, im Inneren der Nordspitze befanden sich zumindest einige Trampelpfade, die erkennen lassen, dass der Bereich bereits zuvor zumindest von Menschen aufgesucht und erforscht worden ist. Die im Rahmen der Baugrunduntersuchung bei den Bohrstellen RSK 17, 18, 20, 21 und 26 gefundenen Aufschüttungen/Auffüllungen belegen zusätzlich den vorhandenen anthropogenen Einfluss in Teilen des Bauabschnitts 1, insbesondere im Bereich der beiden südlichen Spielinseln.

Zusätzlich abgeschwächt wird der Eingriff dadurch, dass die Planung erkennbar bemüht ist, die Spielinseln möglichst in die Natur zu integrieren, insbesondere nur wenige Bäume zu fällen sind. Soweit der Antragsteller geltend gemacht, die zusätzlich durchgeführten Kronenschnitte und etwaige durch die Baumaßnahmen verursachten Schäden an den Wurzeln würden zu weiteren irreversiblen Schäden an den Bäumen führen, ist diese Einschätzung vor dem Hintergrund der von der Beigeladen vorgesehenen Schutzmaßnahmen nicht plausibel.

Auch die Wertigkeit des betroffenen Bereichs ist nicht so hoch einzustufen, dass dies einen Eingriff von vornherein unmöglich machen würde. Der Antragsgegner hat nachvollziehbar dargelegt, dass die fachlichen Voraussetzungen für eine Klassifizierung der Fläche als Auenwald nicht vorlägen. Nach der Kartieranleitung des LANUV seien Waldbestände auch bei regelmäßiger bzw. episodischer Überflutung nicht als Auenwälder zu kartieren, wenn in der Baum- und Strauchschicht der Anteil typischer Auenwaldarten weniger als $70 \%$ betrage und in der Krautschicht Brennnessel Dominanzbestände (Deckung größer $75 \%$ ) und/oder Neophyten (z. B. Imaptiens glandulifera, Deckung $>50 \%$ ) vorherrschen. Dies sei bei der Nordspitze der Fall. Dem ist der Antragsteller nicht substantiiert entgegengetreten.

Dahinstehen lässt die Kammer, ob die vorgesehenen Kompensationsmaßnahmen eingriffsmindernd zu berücksichtigten sind, vgl. zum Habitatschutzrecht BVerwG, Urt. v. 9.7.2009 - 4 C 12.07, juris, Rdnr. 28.

Den durch den Eingriff verursachten Nachteilen stehen öffentliche Interessen von erheblichen Gewicht gegenüber. Die im Bauabschnitt 1 geplanten Wege werden von einer Vielzahl unterschiedlicher Nutzergruppen (Spaziergänger mit oder ohne Hund, Sportler, Familien mit Kindern) genutzt werden und dadurch, wie bereits ausgeführt, den wertvolleren Südostteil der Insel entlasten. Die Spielinseln, deren Flächen einen erheblichen Anteil des Eingriffs ausmachen, sind zwar wohl in erster Linie nur für Familien mit Kindern bzw. ggf. für Jugendliche attraktiv, können also auch nur für diese Nutzergruppe eine relevante Lenkungswirkung entfalten. Familien mit Kinder gehören nach den übereinstimmenden Angaben von Antragsgegner und Beigeladener aber ohnehin schon nicht zu den typischen Nutzern der Südspitze der Insel. Allerdings verfolgt die Beigeladene mit der Schaffung der Spielinseln nicht nur das Ziel, Besucher vom Südostteil der Insel fernzuhalten, sondern sie will gleichzeitig auch das Angebot für Familien mit Kindern verbessern, die Insel zur Erholung zu nutzen,. Dieses Ziel wird durch die geplanten Spielinseln im Vergleich zum bisherigen Zustand erheblich gefördert, weil der bislang vorhandene einzelne Spielplatz veraltet und regelmäßig überfüllt ist. 
Es kann dabei dahinstehen, ob die Nutzung der Spielinseln als Erholung im Sinne des $\$ 3$ Nr. 2 lit. c) LSGVO zu verstehen ist. Nach dieser Vorschrift erfolgte die Unterschutzstellung wegen der besonderen Bedeutung der Landschaftsschutzgebiete für die Erholung, insbesondere für die landschaftsorientierte Naherholung sowie für das Naturund Landschaftserleben in der Nähe städtischer Ballungsgebiete. Würde die Nutzung der Spielinseln dem unterfallen, würde die Errichtung der Spielinseln sogar eines der Ziele der Unterschutzstellung fördern. Aber selbst wenn man die Schaffung von Erholungsmöglichkeiten durch die Errichtung hierzu geeigneter baulicher Anlagen nicht dem landschaftsschutzrechtlichen Erholungsbegriff unterwerfen sollte, vgl. dies ablehnend hinsichtlich des Begriffs der Erholung in $\int 7$ Abs. 1 Nr. 3 BNatSchG: Gellermann, in: Landmann/Rohmer, Stand: 91. EL September 2019, \& 7 BNatSchG, Rdnr. 6; Klages, in: Frenz/Müggenborg, BNatSchG, 2. Aufl. 2016, $\$ 7$ Rdnr. 4, ändert dies nichts daran, dass es sich um ein im öffentlichen Interesse liegendes Ziel handelt, das in die Abwägung einzustellen ist. Im Habitatschutzrecht werden Freizeitbelange zwar als Gemeinwohlbelang minderen Gewichts eingeordnet, der von vorherein Eingriffe in ein FFH-Gebiet nicht rechtfertigen kann, vgl. EuGH, Urt. v. 28. 2. 1991 - C-57/89, Rdnr. 22; BVerwG, Urt. v. 17. 1.2007 - 9 A 20.05, juris, Rdnr. 129;

Da Landschaftsschutzgebiete aber einem deutlich schwächeren Schutz unterliegen, ist dies auf die naturschutzrechtliche Befreiung nach $\$ 67 \mathrm{BNatSchG}$ nicht übertragbar.

Dies zu Grunde gelegt überwiegen die mit dem Vorhaben verfolgten Ziele in ihrer Kombination die Belange des Landschaftsschutzes. Der gewichtige Eingriff in die Nordspitze der Insel ist gerechtfertigt, weil durch die überarbeiteten bzw. neu angelegten Wege verschiedene Nutzergruppen effektiv von den im Vergleich zur Nordspitze schützenswerten Teilen der Insel weggeleitet werden können. Auch die Spielinseln werden zumindest in einem gewissen Ausmaß Besucher binden (und dadurch andere Teile der Insel mittelbar schützen) und verbessern zudem erheblich die Naherholungsmöglichkeiten jedenfalls für Familien mit Kindern auf der Insel.

e) Die von dem Antragsgegner getroffene Ermessensentscheidung ist rechtlich nicht zu beanstanden. Der angegriffene Bescheid kann hinsichtlich des Bauabschnitts 1 aufrechterhalten werden, auch wenn er sich hinsichtlich des Bauabschnitts 2 und 3 als rechtswidrig erweist. Der Verwaltungsakt ist insofern teilbar, weil der Bauabschnitt 1 räumlich klar von den anderen beiden Bauabschnitten abgegrenzt ist und die Maßnahmen in Bauabschnitt 1 auch ohne Verwirklichung der Planung in den Bauabschnitten 2 und 3 sinnvoll bestehen bleiben können. Keine der Maßnahmen in Bauabschnitt 1 setzt voraus, dass auch Bauabschnitt 2 und 3 umgesetzt werden.

f) Das in Bauabschnitt 1 geplante Vorhaben verstößt auch nicht gegen die naturschutzrechtliche EingriffsregeIung.

Nach $\$ 14$ Abs. 1 BNatSchG sind Eingriffe in Natur und Landschaft im Sinne des Bundesnaturschutzgesetzes Veränderungen der Gestalt oder Nutzung von Grundflächen oder Veränderungen des mit der belebten Bodenschicht in Verbindung stehenden Grundwasserspiegels, die die Leistungs- und Funktionsfähigkeit des Naturhaushalts oder das Landschaftsbild erheblich beeinträchtigen können.

Gemäß $\$ 15$ Abs. 1 und Abs. 2 Satz 1 BNatSchG ist der Verursacher eines Eingriffs verpflichtet, vermeidbare Beeinträchtigungen von Natur und Landschaft zu unterlassen, und unvermeidbare Beeinträchtigungen durch Maßnahmen des Naturschutzes und der Landschaftspflege auszugleichen (Ausgleichsmaßnahmen) oder zu ersetzen (Ersatzmaßnahmen). Nach $\$ 15$ Abs. 5 BNatSchG darf ein Eingriff nicht zugelassen oder durchgeführt werden, wenn die Beeinträchtigungen nicht zu vermeiden oder nicht in angemessener Frist auszugleichen oder zu ersetzen sind und die Belange des Naturschutzes und der Landschaftspflege bei der Abwägung aller Anforderungen an Natur und Landschaft anderen Belangen im Range vorgehen.
Der Behörde steht bei der Bewertung der Eingriffswirkungen eines Vorhabens und ebenso bei der Bewertung der Kompensationswirkung von Ausgleichs- und Ersatzmaßnahmen, insbesondere was deren Quantifizierung betrifft, eine naturschutzfachliche Einschätzungsprärogative zu. Die vorgenommenen Quantifizierungen bei Eingriffswirkungen und Kompensationsmaßnahmen sind daher nur einer eingeschränkten gerichtlichen Kontrolle zugänglich; sie sind vom Gericht hinzunehmen, sofern sie im Einzelfall naturschutzfachlich vertretbar sind und auch nicht auf einem Bewertungsverfahren beruhen, das sich als unzulängliches oder gar ungeeignetes Mittel erweist, um den gesetzlichen Anforderungen gerecht $\mathrm{zu}$ werden, vgl. BVerwG, Urt. v. 21.1.2016 - 4 A 5.14, juris, Rdnr. 146, und vom 9. 6.2004 - 9 A 11.03, juris, Rdnr. 118.

Ausgehend hiervon ist nichts dafür ersichtlich, dass die durch den Bauabschnitt 1 bedingten Auswirkungen auf verschiedene Schutzgüter in dem angegriffenen Bescheid und dem Landschaftspflegerischen Begleitplan falsch bewertet worden sind. Die Einwände des Antragstellers greifen nicht durch.

Aus dem Vermeidungsgebot folgt nicht, wie der Antragsteller meint, dass der Eingriff in die Nordspitze zu unterlassen ist, weil die Spielflächen an anderen Orten auf der Insel untergebracht werden können. Dies trifft aus den bereits zur naturschutzrechtlichen Befreiung dargestellten Gründen schon nicht zu, weil die Spielflächen an anderen Stellen auf der Insel die ihnen zugedachten Funktionen weniger stark erfüllen könnten. Unabhängig hiervon zielt das Vermeidungsgebot aber ohnehin nicht auf die Vermeidung des Eingriffs, sondern auf die Vermeidung der mit ihm verbundenen nachteiligen Folgen, vgl. BVerwG, Beschl. v. 19.9. 2014 - 7 B 7.14 -, juris, Rdnr. 15, m.w. N.

Aus $\$ 15$ Abs. 1 Satz 2 BNatSchG (,am gleichen Ort“) ergibt sich, dass insoweit Alternativstandorte nicht zu prüfen sind, vgl. BVerwG, Urt. v. 14.7.2011 - 9 A 12.10, juris, Rdnr. 154; OVG Münster, Urt. v. 29.3.2017 - 11 D 70/09. AK, juris, Rdnr. 1017.

Aus diesem Grund steht das Vermeidungsgebot auch nicht der von der Nordbrücke abgehenden Treppe entgegen. Auch insoweit ist die Einrichtung der Treppe gerade der Eingriff, dessen Existenz vom Vermeidungsgebot vorausgesetzt wird.

Die weiteren Einwände des Antragsgegners zum Vermeidungsgebot betreffen schon überwiegend nicht den Bauabschnitt 1, begründen im Übrigen keine erheblichen Beeinträchtigungen der Schutzgüter und sind derart unsubstantiiert, dass sie nicht zuletzt unter Berücksichtigung der naturschutzfachlichen Einschätzungsprärogative des Antragsgegners dessen Bewertung nicht in Zweifel zu ziehen vermögen.

2. Ist der angegriffene Bescheid hinsichtlich des Bauabschnitts 1 rechtmäßig, überwiegt auch das Vollziehungsinteresse das Aussetzungsinteresse des Antragstellers. Dabei kann dahinstehen, ob die von Antragsgegner und der Beigeladenen im Zeitpunkt der Anordnung der sofortigen Vollziehung geltend gemachten Umstände (bevorstehendes Festival vom 29. Mai bis 7.6.2020, Pachtvertrag über Eröffnung des lnselcafes im Mai, Verlust der eingesetzten Arbeitskräfte im Fall der Aussetzung der Vollziehung, fehlende Möglichkeit bewilligte Fördergelder abzurufen) die Entscheidung heute noch tragen können. Dies ist wegen der durch die Coronavirus Pandemie bedingten Auswirkungen (Absage des Festivals, Einschränkungen in der Gastronomie) und wegen der Wiederherstellung der aufschiebenden Wirkung für einen Teil des Bescheids nicht sicher. Jedenfalls sind aber die in Bauabschnitt 1 geplanten Maßnahmen ausreichend reversibel, sodass keine nachhaltigen Schäden für Natur und Landschaftsschutz zu befürchten sind. Die Kammer nimmt insoweit Bezug auf ihre Ausführungen im Beschl. v. 12.2.2020. 